the extent of their roles in vivo is not yet understood. These complexes are also of interest because they share five subunits and possess histone acetylase activities, raising the possibility that they are functionally redundant. The results of genomewide analysis on individual components of TFIID and SAGA reveal that expression of most genes requires the function of one or more of the shared subunits, demonstrating that SAGA and TFIID are together required for expression of most genes. Most striking was evidence that the different histone acetylase components of SAGA and TFIID are functionally redundant, revealing that half of the genome can be expressed through the function of either complex.

Chakrvarti, Shukti

\section{Ulcerative colitis and Crohn disease: gene expression profiling using oligonucleotide microarrays}

\section{Shukti Chakravarti \& Ian Lawrance}

Departments of M edicine and Genetics, Case Western Reserve University, Cleveland, O hio 44106, USA

Inflammatory bowel disease (IBD) is a progressive, recurrent gastrointestinal disorder influenced by genetic, immunoregulatory and environmental factors and often requiring surgery for treatment. Ulcerative colitis (UC) and Crohn disease (CD), the two most common forms of IBD, have similar demographic and epidemiological features, but differ in intestinal tissue damage and prognosis. UC is associated with mucosal and submucosal injury involving the rectum and adjacent colon, whereas $\mathrm{CD}$ is associated with extensive transmural damage involving any segment of the colon. Both phenotypes are likely the outcome of small changes in expression of many regulatory genes, with consequent changes in expression of genes encoding cellular and extracellular matrix proteins. To understand the overall gene expression and dynamics in UC and CD we have performed expression profiling using oligonucleotide microarrays.

Resected bowel tissue from fresh UC, CD and control (cancer) surgical specimens were used to prepare total RNA. Poly(A) ${ }^{+}$RNA was extracted from pooled total RNA to prepare biotinylated cRNA for hybridization to the Affymetrix HuGene Fl set according to the manufacturer's recommendations. After hybridization, GeneChips were washed in the Fluidics station 400 and scanned in a Hewlett-Packard GeneArray Scanner. Scanned images were analysed using the GeneChip3.1 Analysis Suite.

Probing of the UC target RNA identified fivefold or higher expression of 8 transcripts from HuGeneFLsubA array, 4 from sub B array, 8 from sub C and 29 from sub D array compared with control RNA. Two independent probings showed remarkable reproducibility (33 of 49 overexpressed transcripts in common) between experiments. Furthermore, overexpressed genes included several 'IBDrelated' genes, such as genes encoding proteins that bind HLA class II cis-acting elements, metalloproteinases and collagen types I and IV chains. Additionally, we identified several novel genes as overexpressed or repressed in UC. Hybridization of the CD sample to sub B, C and D arrays indicate overexpression of ten transcripts at fivefold and higher levels. Compared with UC, fewer genes are overexpressed in $\mathrm{CD}$; for example, in the sub D array compared with 29 overexpressed transcripts in $\mathrm{UC}$, only 6 were elevated in $\mathrm{CD}$, of which only 2 were unique to $\mathrm{CD}$. In general, certain immunoregulatory genes, antimicrobial factor encoding genes, extracellular matrix (ECM) modulatory genes and ECM genes show altered expression in $\mathrm{UC}$ and $\mathrm{CD}$. These studies will lead to a fundamental understanding of IBD pathogenicity, differences underlying $\mathrm{UC}$ and $\mathrm{CD}$, and ultimately to better therapeutic approaches.
Chambers, James

\section{The generation of a viral DNA chip for simultaneous expression measurements of all known ORFs in the largest member of the herpesvirus family, human cytomegalovinus}

\author{
James Chambers \\ Invitrogen, 1600 Faraday Ave, Carlsbad, California, USA
}

We describe, for the first time, the generation of a viral DNA chip for simultaneous expression measurements of all known ORFs in the largest member of the herpesvirus family, human cytomegalovirus (HCMV). In this study, an HCMV chip was fabricated and used to characterize the temporal class of viral gene expression. The viral chip is composed of microarrays of viral DNA prepared by robotic deposition of oligonucleotides on glass for all known ORFs in the HCMV genome. Viral gene expression was monitored by hybridization to the oligonucleotide microarrays with fluorescently labelled cDNAs prepared from mockinfected or infected human foreskin fibroblast cells. By using cycloheximide and ganciclovir to block de novo viral protein synthesis and viral DNA replication, respectively, the kinetic classes of array elements were classified. The expression profile of known and many previously uncharacterized ORFs provided a temporal map of immediate-early $(\alpha)$, early $(\beta)$, early-late $(\gamma 1)$ and late $(\gamma 2)$ genes in the entire genome of HCMV. Sequence compositional analysis of the $5^{\prime}$ non-coding DNA sequences of the temporal classes using algorithms that automatically search for defined and recurring motifs in unaligned sequences indicated the presence of candidate regulatory motifs for early, early-late and late genes. These fabricated microarrays of viral DNA allow rapid and parallel gene expression analysis at the whole viral genome level. The viral chip approach coupled with global biochemical and genetic strategies should greatly speed the functional analysis of established as well as newly discovered large viral genomes.

Chen, Yidong

\section{Microarray ratio analysis under noisy background}

\author{
Yidong Chen ${ }^{1}$, Vishnu Kamat', Edward R. Dougherty', \\ Michael L. Bittner', Paul S. M eltzer ${ }^{1} \&$ Jeffery M. Trent ${ }^{1}$
}

${ }^{1}$ Cancer Genetics Branch, National Human Genome Research Institute, National Institutes of Health, Bethesda, M aryland, USA

${ }^{2}$ Computer Assisted M edical Diagnostic Imaging Laboratory, Department of Electrical Engineering, Texas A\&M University, College Station, Texas, USA

To quantitatively analyse gene expression level, two fluorescently labelled RNAs were routinely hybridized to arrayed cDNA probes on a glass slide. Ratios of gene expression levels from two co-hybridized samples were obtained through image segmentation and signal detection methods. In our early report, the expression ratio has been studied via ratio statistics, and the ratio confidence interval has been established so that ratio outliers can be easily identified. Typically, we assume fluorescent background level does not interfere with ratio measurement, which is derived via background subtraction and weak target elimination. Many experimental results, however, suggested that ratios derived from weak targets possess larger variation than those from strong targets. In this study, we propose a new signal and noise interaction model. Under the noisy environment, the ratio statistic will be numerically evaluated and its self-adjusting confidence interval will be introduced. The new confidence interval, which automatically adapts under different signal-to-noise ratios (SNR), provides a better criterion to further 